\title{
Dose All Fetal Intracranial Hemorrhage Has a Bad Prognosis?
}

\author{
Sahar H Abdulghani* \\ Department of Obstetrics and Gynecology, College of Medicine, King Khalid University Hospital, King \\ Saud University, Medical City, Riyadh, Kingdom of Saudi Arabia
}

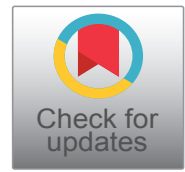

*Corresponding author: Sahar H Abdulghani, MD, Department of Obstetrics and Gynecology, College of Medicine, King Khalid University Hospital, King Saud University, Medical City, P.O BOX 4663, Riyadh 11412 Kingdom of Saudi Arabia, Tel: +966-505256616, E-mail: Sabdulalghani@KSU.EDU.SA; saharhhag@yahoo.com

\begin{abstract}
Fetal intra-cranial hemorrhage $(\mathrm{ICH})$ is a rare complication diagnosed during pregnancy with subsequent fetal neurological sequelae or death. Hemorrhage can occur anyplace in fetal cranium it can take within the subarachnoid, subdural, intraparenchymal place and Intra-ventricular hemorrhage (IVH), which considered the most identified hemorrhage prenatally. Neurodevelopmental prognosis on children varies upon the etiology, the severity of the bleeding causing ventricular dilatation, midline shift and brain insult. Prenatal diagnosis of intra-cranial hemorrhage has been widely reported due to improvement in imaging technology making an effective counseling and management antenatally there is varies predisposing factors, but in some cases the cause remains unidentified. The etiology, timing of occurrence and the severity of fetal $\mathrm{ICH}$ have significant impact on the morbidity and mortality. The author reports case of primigravida in third trimester with fetal $\mathrm{ICH}$ with no obvious determined cause resolved spontaneously in twomonth post-delivery with good fetal outcome without neurological impact.
\end{abstract}

\section{Keywords}

Intracranial, Intraventricular, Hemorrhage, Ultrasound, Magnetic resonance image

\section{Introduction}

Antenatal diagnosis of fetal intracranial hemorrhage (ICH) has been widely reported due to improvement in both sonographic imaging technology and Magnetic resonant image (MRI). Sonographic findings of fetal $\mathrm{ICH}$ are variable and times subtle however, many cases remains unidentified antenatally with difficult to differentiate it from other intracranial lesions [1] with continuous improvement of ultrasound equipment's number of fetal diagnoses is increase on the other hand MRI technology provides highly accurate additional information specifically in those cases where antenatal sonography is inconclusive $[2,3]$.

Fetal hemorrhagic is a pathophysiological processes that lead to antenatal brain insult associated with postnatal neurological squeal e.g. seizure disorders, mental retardation psychomotor delays and cerebral palsy.

$\mathrm{ICH}$ has many types e.g. intra-ventricular hemorrhage, cerebellar hemorrhage and intra-parenchymal hemorrhages. Most of the intracranial hemorrhages are located in the supratentorial area and less frequently at the infratentorial fossa while germinal matrix-intraventricular hemorrhage in the fetus is very rare.

The incidence of $\mathrm{ICH}$ is unclear; but some of authors estimate the actual prevalence of fetal intracranial hemorrhage is approximately between 5 in 10.000 and approximately $25 \%-45 \%$ in premature infants with birth weight less than 1500 grams. In term neonates cortical injury is more common but in premature neonates injury mainly occurs at brain parenchyma in hippocampus, pons and cerebellum and in the periventricular germinal matter causing selective neuronal necrosis, and periventricular leukomalacia.

This form of intracranial bleeding is rare in mature fetuses with few cases have been described. Fetal ICH is rare complication in pregnancy and mostly occurs with predisposing factors the pathogenesis of $\mathrm{ICH}$ is believed to be multifactorial e.g. genetic, maternal, fetal and placental cause while in the majority of cases no identifiable risk factor is found.

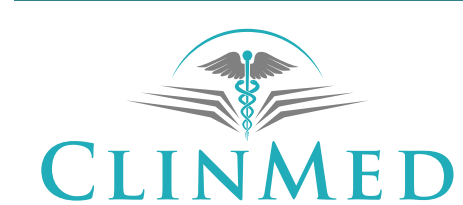

INTERNATIONAL LIBRARY
Citation: Abdulghani SH (2018) Dose All Fetal Intracranial Hemorrhage Has a Bad Prognosis?. Int J Womens Health Wellness 4:072. doi.org/10.23937/2474-1353/1510072

Accepted: June 04, 2018: Published: June 06, 2018

Copyright: (c) 2018 Abdulghani SH. This is an open-access article distributed under the terms of the Creative Commons Attribution License, which permits unrestricted use, distribution, and reproduction in any medium, provided the original author and source are credited. 
Maternal risk factors contributing in fetal $\mathrm{ICH}$ includes platelet or coagulation disorder [4] seizures disorder, pancreatitis, cholestasis, Pre-eclampsia, febrile infection [5] Procedure like amniocentesis, maternal trauma [6] platelets function altering.

Medications such non-steroidal anti-inflammatory drugs, warfarin or cholestyramine and cocaine abuse patient. Fetal factors causing intracranial hemorrhage includes congenital coagulopathy bleeding disorder Factor $\mathrm{V}$ and factor $\mathrm{X}$ deficiency, hemorrhage into a congenital tumor, Twin-Twin transfusion syndrome, demise of a co-twin, and fetomaternal hemorrhage. Prognosis varies based on several factors the etiology, location [6] and gestational age of occurrence.

Intracranial hemorrhage in utero has been reported to be a cause of hydrocephaly, non-immune hydrops with severe fetal anemia including the potential for development of hydrocephaly and neurodevelopmental delay. Neurodevelopmental outcome ranges from hemorrhage, absorption and resolution without residual deficit, to brain damage that leads to neurological and mental deficits, epilepsy in extreme cases that may lead to either fetal or neonatal death.

There is no treatment for fetal intracranial lesion has been established and no data indicate that cesarean delivery as a mode of delivery may improve fetal outcome.

Optimal mode of delivery of infant with sonographic evidence of an ICH is uncertain [7]. Severe lesions usually have a poor prognosis and conservative management may be offered.

The author reports a case of fetal intracranial hemorrhage diagnosed at 36 week of gestation for primigravida confirmed postnatally with both neonatal head ultrasound and CT scan. Subsequent head CT scan repeated as a follow up which showed dramatic improvement and regression of the $\mathrm{ICH}$ with normal clinic examination and no brain squeal. A 38-year-old, Gravida 1 known case of Essential Hypertension on medication and Aspirin. She was diagnosed with gestational diabetes as well her blood sugar readings values were under control on diabetic diet. There was no history of trauma or fall during or infection during her pregnancy. Her antenatal screening test was all in normal values. TORCH panel was non-reactive. Blood group is $\mathrm{O}$ positive with negative antibodies. Her first fetal ultrasound done at 22 weeks and 2 days of gestation with normal morphology and fetal biometry. A follow up fetal wellbeing ultrasound repeated at 36 weeks of gestation with the following findings, single viable fetus, cephalic in presentation, normal growth for gestational age. Amniotic fluid volume polyhydraminous Amniotic Fluid Index $33.8 \mathrm{~cm}$. The abnormal finding in fetal head a noted presence of a mixed echogenic intracranial mass, measuring $4 \times 7 \mathrm{~cm}$ with irregular margins occupying the left temporoparietal lobe compressing the posterior horn of left lateral

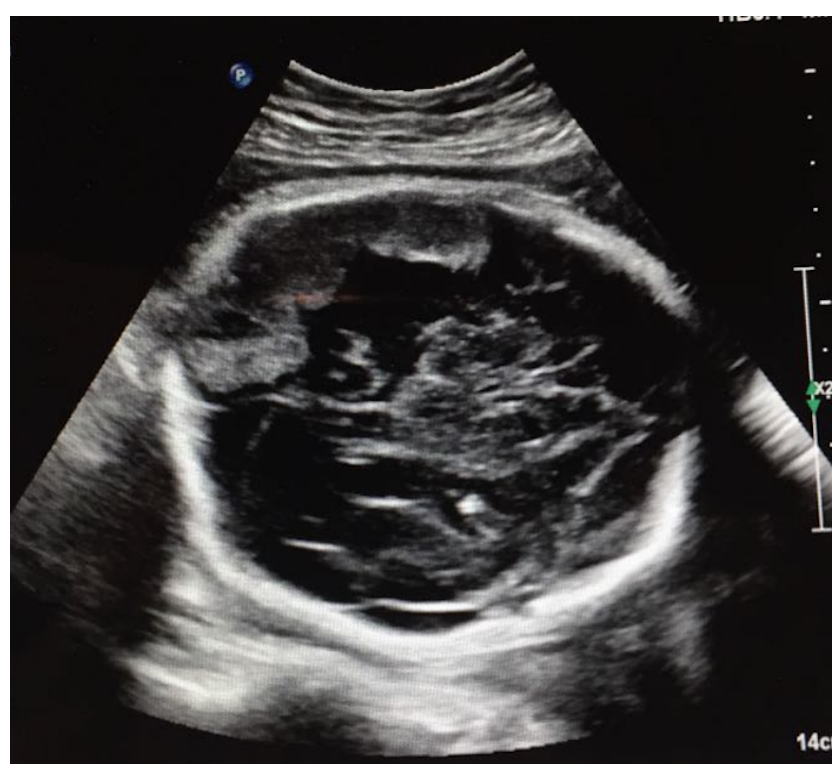

Figure 1: Sonographic demonstration of intracranial hemorrhage at 36 weeks of gestation, an axial view of fetal head showing a mixed echogenic intracranial collection measuring $4 \times 7 \mathrm{~cm}$ with irregular margins occupying at the left temporo-parietal lobe compressing the posterior horn of left lateral ventricle with no element of midline shift.

ventricle with no element of midline shift mild bilateral ventriculomegaly noted. No other structural anomaly noted and nor element of fetal hydropes (Figure 1).

Patient was informed about the ultrasound findings at the high-risk clinic all the possible reasons causing fetal intra-cranial hemorrhage was explained, antenatal management by close monitoring with elective plane of delivery was discussed. Multidisciplinary team will be involved post-delivery e.g. pediatric neurology, surgery and hematology and neonatology. She was explained that prognosis of fetal intracranial bleeding varies based on the degree of severity affecting adjacent brain tissues, it is also depend on the underlying etiology and the timing it occur. Patient was admitted at 37 week for planned caesarean delivery due to premature contraction caused by the polyhydraminous.

A complete work up was requested to exclude fetal-neonatal alloimmune thrombocytopenia (FNAIT) and maternal blood work up was all requested and result came to be within normal. Neonatology team consulted earlier for post-delivery plan of management. Patient underwent an elective caesarean delivery with good outcome of a healthy baby boy. Cried immediately, pink in color, Apgar 9/1 min and 9/5 min Heart Rate: 141 bpm Respiratory rate 40 Tem: 36.4 Baby delivered in a good general condition with no evidence of hydrocephalous. There was mild subcostal retraction for which plane to be admitted in the NICU for Observation and work up. Central nervous system examination was with normal finding. Ophthalmic examination with normal retina and no evidence of hemorrhage or papilledema. 


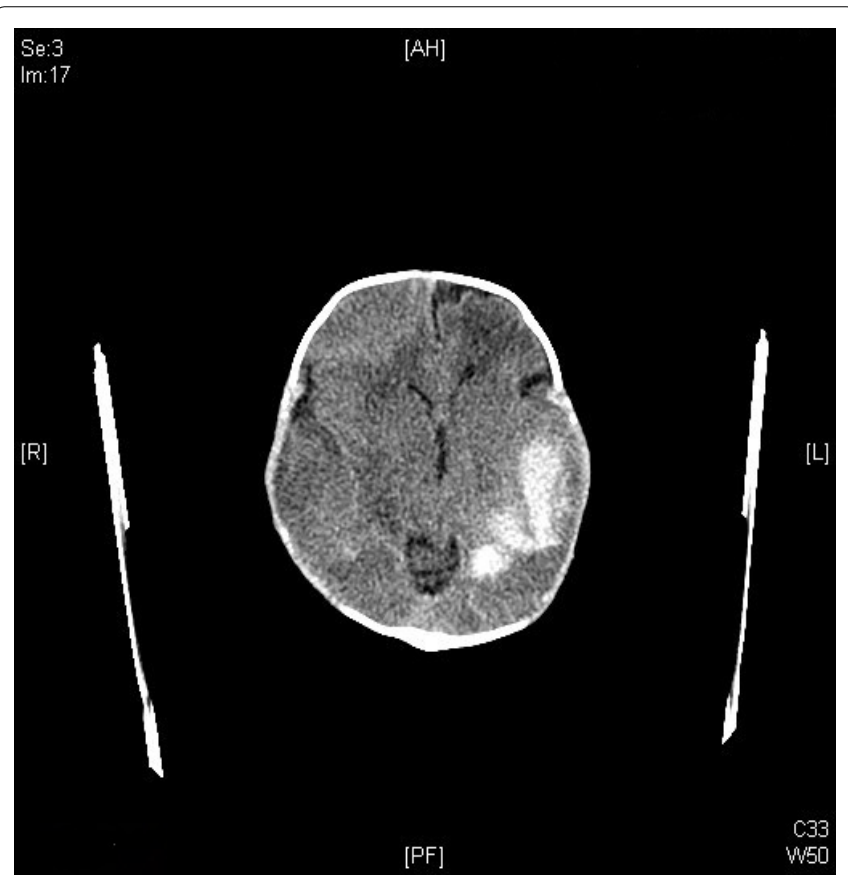

Figure 2: CT image of infant head, a coronal view demonstrate large intraparenchymal hemorrhage located at the left temporo-parietal lobes diffuse hypodensities of cerebral parenchymal.

\section{Labs investigation}

Blood group O Positive.

WBC: 21.400

HB: 122.0

HCT: 38.2

Platelet count: 480

PT: (59.5 High) initially then dropped to 17.7 and then to 15.00

APTT: (90.60 High) dropped 55.90 then 42.40

INR: (6.19 High) then normalized to 1.34

LFT, Electrolytes and blood gases results were all within the normal range.

Due to prolonged initial result for coagulation profile, the plan was by the NICU team to Perform bleeding screening test PT/APTT, INR Fibrinogen level, coagulation factors assay and to consider PRBC transfusion if there is significant drop in the HB value.

FFP $10 \mathrm{ml} / \mathrm{kg}$ Q12 HRS if coagulation values still elevated and vitamin $\mathrm{K} 1 \mathrm{mg}, 0.1 \mathrm{ml} \mathrm{IM}$ given once.

Blood, urine culture and metabolic panel were all requested with negative result.

\section{Head ultrasound}

Intra-parenchymal hemorrhage in left cerebral hemisphere with no minimal midline shift. A mild ventricular dilatation on the left side noted.

\section{CT brain}

A large intraparenchymal hemorrhage in the left temporoparietal lobes, diffuse hypodensities of cerebral parenchymal. Multiple extra-axial fluid collection likely subacute hemorrhage along convexity of both cerebral hemispheres (Figure 2).

The initial coagulation profile results post-delivery was high then it got normalized in the repeated subsequent test it was noted that blood clotting factor 11 was initially prolonged then it got normalized in subsequent testing which raised the suspicion of possible cause of fetal intracranial hemorrhage.

Pediatric hematology team consulted who excluded factor 11 as a cause of fetal intracranial hemorrhage advising for repeating $\mathrm{CT}$ head with angio to rule out congenital arterial venous malformation.

The principle in management fetal ICH at our organization requires admission to the NICU initially and stabilization of the newborn general condition.

A multidisciplinary team will be involved including a pediatric hematologist Neurosurgeon, neurologist, ophthalmologist and a pediatric radiologist.

A Complete blood work-up will be ordered and followed regularly based on the initial test results. Pediatric hematology team will be consulted and will be involved in terms of management e.g. Vitamin $\mathrm{K}$ deficiency, Alloimmune Thrombocytopenia, and other Congenital hematological bleeding disorders.

Treatment modality varies based on the blood test results, including blood transfusion and replacement of blood products.

Radiology investigation will be initially done by head ultrasound to rule out brain tissue masses, Arterial-Venous malformation $(A-V)$ and other possible causes. The exact size, location and the extent of the bleeding will be followed by the Neurosurgery team to decide for the need of any surgical intervention.

CT head will be arranged after the ultrasound result but other radiological testing like MRI or MRV will be arranged per case based on the possible diagnosis or in case if the Initial testing were inconclusive. Repeat radiological test will be performed as a follow up in two to three month intervals to evaluate the progression of the hemorrhage. Upon discharging follow-up will be given to the high-risk pediatric Clinic for both Sensory and motor function evaluation by the pediatric neurology team.

Genetic testing will be offered in case of suspected or diagnosed familial inherited bleeding disorders.

Other possible causes of $\mathrm{ICH}$ are excluded initially from detailed maternal history and work-up like Infection, trauma and drug intake.

Total hospital stay in the NICU was for 8 days baby was discharged home for conservative management as an out Patient in a good neurological and general 


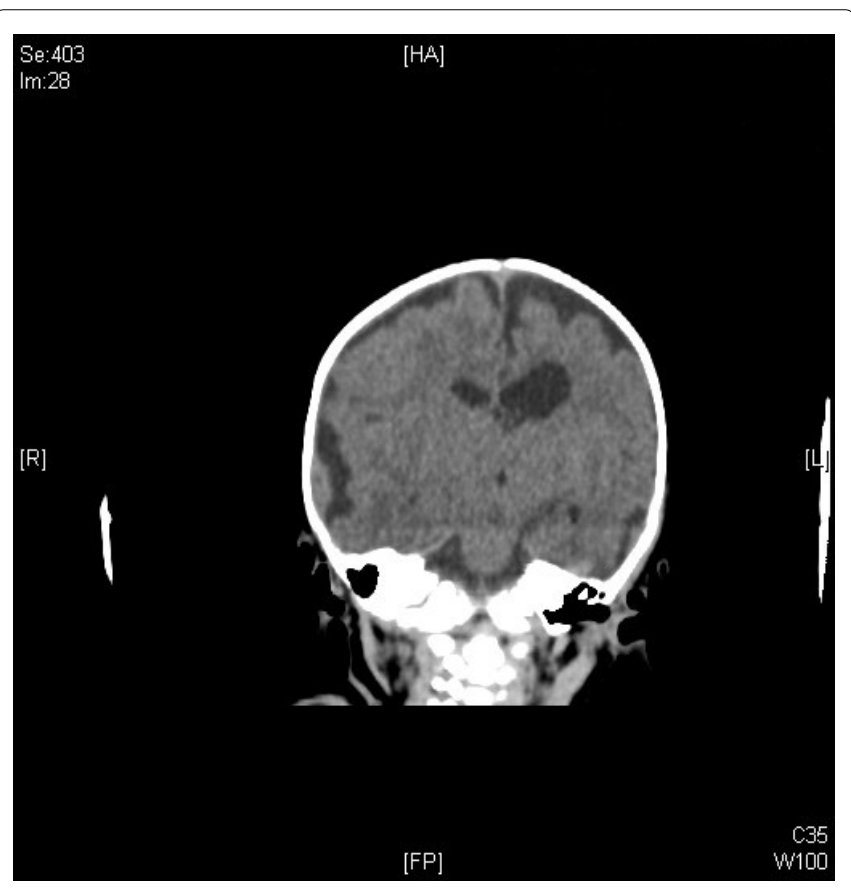

Figure 3: CT head at 2 month of age, coronal view demonstrate a marked regression of the subacute hemorrhage.

condition. A repeated CT head at the age of 2 month of age Figure 3 showed a marked interval regression of the previously seen multiple extra-axial fluid collection (sub-acute hemorrhage) with multiple area of residual $0.4 \mathrm{~cm}$ at the temporal $1 \mathrm{~cm}$ at the left parietal and 0.2 $\mathrm{cm}$ at the right frontal region.

No midline shift, no acute territorial infarction, sutures appears unremarkable with no fractures (Figure 3).

Subsequent visit at the age of 3 month and then at the age of 5 month in which clinical examination done in general was unremarkable with no evidence of neurodevelopmental delay neither brain insult.

\section{Discussion}

There is limited data on neurodevelopmental prognosis and their long-term future outcome with different types of fetal ICH. T. Ghi, et al. [1] done extensive literature review of 93 cases reported a poor prognosis of newborns whom diagnosed antenatally with $\mathrm{ICH}, 40 \%$ of fetuses dying in utero or within the first month after delivery and about fewer than half of the survivors will have neurodevelopmental normal at short-term follow-up. The extent of intraventricular hemorrhage varies a lower grade (I-II) with complete disappearance of sonographic findings is associated with a better postnatal outcome and a higher grade intraventricular hemorrhage is associated with a worse neurodevelopmental prognosis.

U. elchalal, et al. [7] concluded antenatal sonographic diagnosis of fetal stroke with IVH Grade III-IV or with brain parenchymal involvement are associated with poor neurological outcome. Gould, et al. in 2005 [8] was first to report COL4A1 mutations in mice with perinatal hemorrhage and in human families with porencephaly.
This mutations have been reported association with a wide spectrum of symptoms, including familial porencephaly, hemorrhagic stroke, infantile hemiparesis and intra-ventricular hemorrhage in preterm infants with parenchymal hemorrhage of antenatal onset, intracranial aneurysms. More extensive supratentorial lesions, in combination with cerebellar injury have recently been reported making this diagnosis has important implications for perinatal management and genetic counseling.

In this case report, the transient elevation of coagulation profile was managed post-delivery with one dose of FFP $10 \mathrm{ml} / \mathrm{kg}$ and Vitamin $\mathrm{K} 1 \mathrm{mg}, 0.1 \mathrm{ml} / \mathrm{IM}$ with no obvious cause explain the elevation of coagulation profile values, most of the work up were within normal values leaving no actual reason found causing $\mathrm{ICH}$. Won Seok Lee, et al. [9] present a case of severe intracerebral and intraventricular hemorrhage in a neonate with congenital FVII deficiency, which is a rare autosomal-recessive bleeding disorder. Clinical findings vary ranging from asymptomatic to life threatening bleeding.

Life-threatening hemorrhaging is rare in about $5 \%$ of the bleeds and occurs most frequently during the first six months of life. Treatment protocol involved FVII replacement therapy using fresh frozen plasma (FFP) prothrombin complex concentrates (PCCs) or plasma FVII concentrates.

In conclusion ICH is the leading cause of neonatal morbidity and mortality underlying Etiology could be either inherited or acquired causes and the clinical outcomes of $\mathrm{ICH}$ varies with the maturity of the brain, underlying etiology, location and the extent of the lesion as the presence of other concurrent disorders [10]. Neurological assessment of delay brain insult needed to be followed with MRI which is a technique has an advantage for ability to distinguish hemorrhage of different ages utilizing the differing magnetic properties of aging blood products. MRI is also superior to CT in soft tissue resolution allowing for assessment of white matter and grey matter lesions alike. MRV is a modality of testing to rule out fistulas or $\mathrm{A}-\mathrm{V}$ malformation.

Late third trimester manifestation of acute fetal $\mathrm{ICH}$ identified antenatally with no midline brain shift and no ventricular enlargement has less effect squeal in the brain and good future neurological prognosis, blood work up to newborn should include coagulation profile and platelet count. Congenital bleeding disorder secondary due to deficiency of one of the clotting factor should be excluded for early diagnose and management. Unidentified obvious cause of fetal ICH requires genetic testing including testing the couple that may add in future subsequent pregnancies. Regular clinical evaluation and radiological testing by MRI or MRA is essential in assessment of long Term neurologically impact of intracranial hemorrhage. 


\section{Conflicts of Interest Statement}

There is no actual or potential, commercial or academic conflict of interest to declare in relation to this study.

\section{Acknowledgment}

The author would like to thank both of Dr. Yasser Amer and Dr. Yasser Saber for their support and contribution during the writing up of this case report.

\section{Funding}

There was no funding applicable for this study.

\section{References}

1. Ghi T, Simonazzi G, Perolo A, Savelli L, Sandri F, et al. (2003) Outcome of antenatally diagnosed intracranial hemorrhage: Case series and review of the literature. Ultrasound Obstet Gynecol 22: 121-130.

2. Gorincour G, Rypens F, Lapierre C, Costa T, Audibert F, et al. (2005) Fetal magnetic resonance imaging in the prenatal diagnosis of cerebellar hemorrhage. Ultrasound Obstet Gynecol 27: 78-80.

3. Malinger G, Lev D, Lerman-Sagie T (2002) Is fetal magnetic resonance imaging superior to neurosonography for detection of brain anomalies? Ultrasound Obstet Gynecol 20: 317-321.
4. (1999) ACOG practice bulletin: Thrombocytopenia in pregnancy. Number 6, September 1999. Clinical management guidelines for obstetrician - gynecologists. American College of Obstetricians and Gynecologists. Int J Gynaecol Obstet 67: 117-128.

5. Moinuddin A, McKinstry R, Martin K, Neil J (2003) Intracranial hemorrhage progressing to porencephaly as a result of congenitally acquired cytomegalovirus infection--an illustrative report. Prenat Diagn 23: 797-800.

6. Strigini F, Cioni G, Canapicchi R, Nardini V, Capriello P, et al. (2001) Fetal intracranial hemorrhage: Is minor maternal trauma a possible pathogenetic factor? Ultrasound Obstet Gynecol 18: 335-342.

7. Elchalal U, Yagel S, Gomori J, Porat S, Beni-Adani L, et al. (2005) Fetal intracranial hemorrhage (fetal stroke): Does grade matter? Ultrasound Obstet Gynecol 26: 233-243.

8. Gould DB, Phalan FC, Breedveld GJ, van Mil SE, Smith RS, et al. (2005) Mutations in Col4a1 cause perinatal cerebral hemorrhage and porencephaly. Science 308: 1167-1171.

9. Lee W, Park $Y$ (2010) A case of intracranial hemorrhage in a neonate with congenital factor VII deficiency. Korean $\mathrm{J}$ Pediatr 53: 913-916.

10. Tan AP, Svrckova P, Cowan F, Chong WK, Mankad K (2018) Intracranial hemorrhage in neonates: A review of etiologies, patterns and predicted clinical outcomes. Eur $\mathrm{J}$ Paediatr Neurol. 